\title{
Application of Newton's method to solve optimization geodetic tasks
}

\author{
D. A. Bykasov,A. V. Zubov, M. G. Mustafin
}

Saint Petersburg Mining University, Department of engineering geodesy, Saint Petersburg, Russian Federation

\begin{abstract}
The article provides general information on nonlinear methods of programming. The algorithm for determining the minimum of various target functions by Newton's method of the second order is considered. The article presents the main advantages and disadvantages of the method. A circular approximation of the chimney measurement results is performed using Newton's method. The algorithm for solving these tasks was implemented in the Visual Basic for Applications software environment. The comparative analysis was held based on Newton's method of the second order with the gradient methods for solving engineering and geodetic tasks. The data obtained allow considering the possibility of the further application of Newton's method in geodesy, especially while solving nonlinear optimization problems. The development of modern technology allows to automate the computing process by writing specific software modules. This makes the process of calculating parameters convenient for the surveyor, as it relieves them from routine calculations.
\end{abstract}

\section{GENERAL INFORMATION ON OPTIMIZATION METHODS IN GEODESY}

The role and importance of engineering geodesy in the 21 st century keep growing, the new tasks are emerging that require different approaches for finding their solutions. The issue of optimization of geodetic calculations and measurements is particularly relevant. In solving some tasks, the speed and accuracy of obtaining the desired parameters, as well as the automation of the solutions, play a special role. In its turn, the development of geodetic instruments has made it possible to solve many geodetic problems at a new technological level. So in his works, $[1,2]$ used satellite and laser equipment to solve classical geodetic problems. Thanks to modern scanning systems, robotic total stations, a surveyor can obtain a huge array of data about the object in a short period. The presence of a large amount of source data allows calculating the desired parameters many times, which increases the reliability of the data. From a large number of solutions, the surveyor needs to choose the optimum correct result. Thanks to the development of information technologies and the introduction of programming methods in geodetic production, it is possible to solve many problems using new methods; this idea is confirmed in the articles [3, 4]. This allows to consider the relevance of the application of optimization methods when solving geodetic tasks.

In a broader sense, optimization should be understood as the activities aimed at obtaining the best result under certain conditions (maximizing profit, minimizing costs). The theory 
of optimization methods is not new; Isaac Newton and Karl Gauss in the 18th century were engaged in the development of a theory for finding optimal solutions to applied problems. They formulated the fundamentals of optimization theory. There are a huge number of optimization methods: methods based on the use of Lagrange multipliers, methods of dynamic programming, and methods of the calculus of variations, linear and nonlinear programming methods. At present, optimization problems exist in various fields of geodesy. It is not possible to choose one optimization method that would solve all problems in modern geodesy without exception. Many optimization methods have been specifically developed for mathematical models of a certain kind. For example, if the original objective function $f(x)$ is expressed by a nonlinear dependence, it is recommended to use the nonlinear programming methods. Dynamic programming in geodesy is used if complex processes that change over time occur when searching for the minimum or maximum of the objective function $f(x)$. Linear programming methods are used when the original objective function $f(x)$ is the sum of linear functions. Therefore, studying the possibility of using different methods to solve problems of a particular group is the best way to choose an optimization method to solve a specific task.

A large class of tasks is associated with processing and obtaining the desired parameters by solving non-linear equations: finding communication parameters between different coordinate systems, designing and adjusting geodetic networks, building digital terrain models, and predicting deformations. All of the above tasks are related to solving nonlinear equations, as well as processing a huge amount of source data.

The widespread introduction of computers made it possible to apply the theory of optimization in solving applied problems. It was possible to widely implement optimization methods in geodetic production and automation of their algorithm. However, is impossible to choose one method that would solve all problems in modern geodesy without exception. The article studies the possibility of using nonlinear programming methods in geodesy, using the example of solving the problem of approximating the results of chimney circumferential measurements. The determination and prediction of deformations are also an important aspect of geodetic production. In solving these problems, nonlinear equations are used, and methods for determining deformation are considered in the works $[5,6]$. This topic is also reviewed in the works of prominent scientists: $[7,8,9,10,11]$.

Practical application of nonlinear programming methods in geodetic practice has been studied rather poorly, which is associated with a rather long introduction of personal computers in the process of geodetic calculations. The study of methods of this group is an urgent task, as it allows to optimize a large class of geodetic tasks. In the course of the study, the problem of determining the parameters of the chimney section was solved by two methods of nonlinear programming (Newton's second-order method and the gradient descent method with a constant step). The second order Newton's method was chosen as the main one for solving this nonlinear problem. This was done for the following reasons: this method has a strict formula, in the search process, the iteration step is determined using the Hessian matrix (matrix of second-order partial derivatives); the use of the second-order derivatives of the objective function allows to increase the speed of finding a solution. Since the possibility of applying the second-order Newton's method has not been studied well enough, it was decided to choose this method as the main one for solving this production geodetic problem. Below, the main advantages and disadvantages of the chosen method will be presented in detail, as well as the main formula of the method. 


\section{SECOND-ORDER NEWTON METHOD}

The second-order Newton's method belongs to the group of nonlinear programming methods, the implementation of which requires information about the value of the function at a given point, as well as the first and second derivatives. The method is well studied in optimization theory thanks to [8], but its applicability to other fields of science, in particular to applied geodesy, has not been studied sufficiently. Traditionally, optimization problems in geodesy are solved according to the following algorithm: the initial values of unknown quantities (parameters) are specified; and these parameters are related by the equations, with all measured values. The equations are then linearized (for nonlinear problems) by expanding the objective function into a Taylor series and solved together under additional constraining conditions, such as the least-squares method, finding corrections to the preliminary values of the parameters. The theory of correction is discussed in detail by [12].

To derive the main formula for the method by which the iterative process is performed, it is necessary to expand the original objective function $f(x)$ in a Taylor series according to formula (1):

$$
f(x) \approx f\left(x_{k}\right)+f^{\prime}\left(x_{k}\right) \cdot\left(x-x_{k}\right)+\frac{1}{2} f^{\prime \prime}\left(x_{k}\right) \cdot\left(x-x_{k}\right)^{2}
$$

where $f^{\prime}\left(x_{k}\right)=$ the value of the first derivative of the function $f(x)$ at the point $x_{k}$; $f^{\prime \prime}\left(x_{k}\right)=$ the value of the second derivative of the function $f(x)$ at the point $x_{k}$; and $x_{k}$ $=$ approximation to the minimum point.

The second-order Newton method is based on a quadratic approximation of the function, therefore, in the Taylor series, the first three terms must be taken into account to derive an iterative formula. Having received the value $x_{k}$, we can calculate the following approximation $x_{k+1}$ to the minimum point. By substituting in formula (1) $x$ by $x_{k+1}$, and also by denoting the $\Delta x=x_{k+1}-x_{k}$, we can obtain formula (2):

$$
f\left(x_{k+1}\right) \approx f\left(x_{k}\right)+f^{\prime}\left(x_{k}\right) \cdot \Delta x_{k}+\frac{1}{2} f^{\prime \prime}\left(x_{k}\right) \cdot \Delta x_{k}^{2}
$$

Expressing the variable $x_{k+1}$ from formula (2), we can obtain the basic formula of the second-order Newton method (3):

$$
x_{k+1}=x_{k}-H^{-1} \cdot G
$$

where $G=$ matrix of the first derivatives of the function $f(x) ; H=$ matrix of the second partial derivatives of the function $f(x)$ (Hessian matrix); $x_{k}=$ vector column of the determined parameters in the $k$ approximation; $n x_{k}+1=$ vector column of the determined parameters in the $k+1$ approximation. The Hessian matrix is responsible for the rate of convergence and the direction of searching for the extremum point, which is calculated by formula (4): 


$$
H(f)=\left(\begin{array}{cccc}
\frac{\partial^{2} f}{\partial x_{1}^{2}} & \frac{\partial^{2} f}{\partial x_{1} \partial x_{2}} & \mathrm{~L} & \frac{\partial^{2} f}{\partial x_{1} \partial x_{q}} \\
\frac{\partial^{2} f}{\partial x_{2} \partial x_{1}} & \frac{\partial^{2} f}{\partial x_{2}^{2}} & \mathrm{~L} & \frac{\partial^{2} f}{\partial x_{2} \partial x_{q}} \\
\mathrm{~L} & \mathrm{~L} & \mathrm{O} & \\
\frac{\partial^{2} f}{\partial x_{q} \partial x_{1}} & \frac{\partial^{2} f}{\partial x_{2} \partial x_{q}} & \mathrm{~L} & \frac{\partial^{2} f}{\partial x_{q}^{2}}
\end{array}\right)
$$

The main drawback of the second-order Newton method is considered to be the calculation of the Hessian matrix, therefore this method is not widely used in applied geodesy since the calculation of the matrix of second partial derivatives is a rather complicated process. In this regard, some authors [13] when solving optimizing geodetic tasks, tend to use direct methods of search (search methods that don't use derivatives in the iterative process). The advent of personal computers made it possible to automate the process of calculating the Hessian matrix. The calculation of partial derivatives can be implemented in one of the programming languages by a numerical method. Due to this, the task of calculating partial derivatives for any objective function can be fully automated.

At each iteration, it is necessary to determine the sign of the Hessian matrix. The matrix of the second partial derivatives at each iteration should be positive definite, i.e., $H>0$, and only if this condition is met, the direction of the search will lead to a decrease in the objective function $f(x)$. The main advantages of the second-order Newton method are that if the function is quadratic, then iteration is necessary to find the minimum of the objective function $f(x)$ for any value of the starting point. Also, the use of second partial derivatives in the iterative process allows increasing the rate of convergence, as well as increasing the accuracy of the results. Most importantly, this method is less sensitive to the choice of the initial parameter value than first-order methods (using only the first derivatives).

If the objective function $f(x)$ is not quadratic, then $\mathrm{k}$ iterations are necessary to reach the extremum point until the condition for stopping the iterative process is fulfilled. The stopping criterion is usually two parameters. This is either the modulus of the difference in the parameter values at the next iteration relative to the previous one, which must be less than a predetermined number $\mu$. Often this number is associated with the accuracy of the results, which is incorrect. Or it is the value of the function $f(x)$ at the point $x_{k+1}$, which must also be less than the predetermined number $\mu$.

For comparison with Newton's method was chosen method of gradient descent with a constant pitch. This method uses information about the value of the function and the value of the first derivative of the original objective function $f(x)$ to find the extremum point. The main formula of the method can be obtained by equating the third term of the Taylor series to zero in formula (2) and expressing the variable $x_{k+1}$. In matrix form, the iterative formula of the gradient method with a constant step has the following form:

$$
x_{k+1}=x_{k}-q \cdot G
$$

where $q_{k}=$ step size of the iterative process.

The iteration step $q$ affects the convergence rate and the accuracy of the data obtained. Often, if the function is simple, the user chooses the step size based on the condition that the step length from the current point to the new point should be no more than the desired 
accuracy. For example, striving for accuracy of $0.001 \mathrm{~m}$ it is necessary to take a step length of no more than $0.0005 \mathrm{~m}$.

The gradient descent method was chosen for comparison, since only the value of the first derivative of the function is used to find the minimum, so the method is easy to implement. In addition, this method has found wide application in geodetic calculations, as it allows to solve many problems in geodesy.

\section{APPLICATION OF THE METHOD IN PRACTICE}

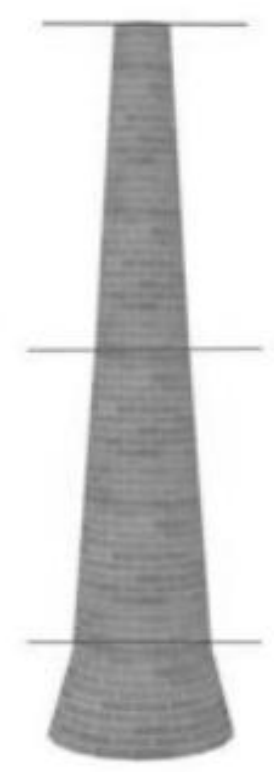

Fig. 1. Chimney.

Let's consider the possibility of using the second-order method of Newton's algorithm, on the example of solving a particular task. We are dealing with the problem of approximating the circumference of the results of measurements of the chimney (Figure 1).

Cross-sectional point appromationis used in various applications using digital image processing methods: [14] used it to determine geometric primitives shown in raster images; [15] investigated its use for diagnosing human vision; [16] used it in various robotic systems for measuring the diameters and lengths of various industrial objects. The parameters of the circle (the coordinates of the center of the circle and the radius) can be calculated in different ways, based on the mapping of characteristic points of the arc, approximation by a parabola, or finding the minimum of various criterial objective functions. To approximate the circumference of the results of measurements of the chimney, it is necessary to determine the coordinates of the center of the circle $X_{0}, Y_{0}$ and the radius $R$. The initial data for solving the problem are the coordinates of the chimney points obtained during the scan. For an unambiguous determination of the section parameters, it is necessary to know the coordinates of three points of the circle. To increase the accuracy of the circle parameters calculation, the coordinates of a larger number of 
points on the circle are determined. When the number of points is more than three, then there is a problem of optimizinge the solution. To determine the parameters of the crosssection, the objective function was created:

$$
f\left(X_{0}, Y_{0}, R\right)=\sum\left(R^{2}-\left(\left(X_{i}-X_{0}\right)^{2}+\left(Y_{i}-Y_{0}\right)^{2}\right)\right)^{2}
$$

where $X_{i}, Y_{i}=$ coordinates of the $i$ - point obtained as a result of scanning, $X_{0}, Y_{0}=$ coordinates of the center of the circle, $R$ = radius of the circle.

As preliminary coordinates of the center of the circle, the average values of the coordinates of the measured points can be taken. The preliminary value of the radius is usually approximately known or it can be calculated from the preliminary coordinates of the center of the circle.

Applying formula (3) to the objective function (6), an iterative process was performed according to second-order Newton method, the obtained values are presented in Table 1. The stopping criterion $\mu$ was $0.001 \mathrm{~m}$.

Table 1. Calculation data of the circle parameters by the Newton method.

\begin{tabular}{lllll}
\hline Iteration & $X_{0} \mathrm{~m}$ & $Y_{0} \mathrm{~m}$ & $R \mathrm{~m}$ & $f\left(X_{0}, Y_{0}, R\right)$ \\
\hline 0 & 702.0000 & 748.0000 & 5.0000 & $2.442 \mathrm{E}+003$ \\
1 & 703.5088 & 749.9764 & 6.6215 & $308.261 \mathrm{E}+000$ \\
2 & 703.2121 & 749.9441 & 6.7899 & $114.743 \mathrm{E}-003$ \\
3 & 703.2157 & 749.9433 & 6.7886 & $82.243 \mathrm{E}-003$ \\
4 & 703.2157 & 749.9433 & 6.7886 & $82.243 \mathrm{E}-003$ \\
\hline
\end{tabular}

The circle parameters were obtained in three approximations. For comparison of the second order Newton method, the problem was solved by using the gradient descent method with a constant pitch. As mentioned above, this method has found wide application in solving geodetic optimization problems; e.g., [11] applied it. The main idea of the method is considered in detail by [16]. The problem was solved in the environment of MathCAD 15. Data for comparison of the second-order Newton method and gradient descent method with a constant pitch are shown in Table 2. For the gradient method, the value $\mu$ was set equal to $0.01 \mathrm{~m}$., since the method did not converge at a value of $0.001 \mathrm{~m}$.

Table 2. The Newton and the gradient methods comparison.

\begin{tabular}{lll}
\hline Parameter & \multicolumn{2}{c}{ Method } \\
\cline { 2 - 3 } & Newton method & Gradient method \\
\hline Initial value ${ }^{\prime}, \mathrm{m}$ & 702.0000 & 702.0000 \\
Initial value $Y_{0}, \mathrm{~m}$ & 748.0000 & 748.0000 \\
Initial value $R, \mathrm{~m}$ & 5.0000 & 5.0000 \\
Final value $X_{0}, \mathrm{~m}$ & 703.2157 & 702.5848 \\
Final value $Y_{0}, \mathrm{~m}$ & 749.9433 & 749.9465 \\
Final value $R, \mathrm{~m}$ & 6.7886 & 6.2397 \\
Number of iterations & 3 & 489 \\
Function value $f\left(X_{0}, Y_{0}, R\right)$ & $82.243 \mathrm{E}-003$ & $8.233 \mathrm{E}+000$ \\
\hline
\end{tabular}

According to the data presented in Table 2, it can be concluded that Newton method is more efficient compared to the gradient descent method with a constant step: using the 
gradient descent method, it is necessary to perform a larger number of iterations to find the minimum compared with the Newton method. According to the value of the objective function $f\left(X_{0}, Y_{0}, R\right)$, we can assume that the local minimum of the function, was found by the gradient method but not the global one.

To automate the process of calculating the circumference parameters, the "Sectional Parameters" a program was created in the Visual Basic for Applications environment. The program can be applied in practice when solving surveying and geodetic problems in determining the rolls (alignments) of cylindrical (conical) objects: chimneys, kilns, tanks, pile drivers, shafts. To run the program it is necessary to enter or copy from a file the coordinates of the points obtained during the measurements in the fields of the MS Office Excel table. The result of the program is MessageBox with the numerical values of the circumferences, which were calculated by second-order Newton method. The visualization of the final result is provided in accordance with the data obtained (Figure 2).

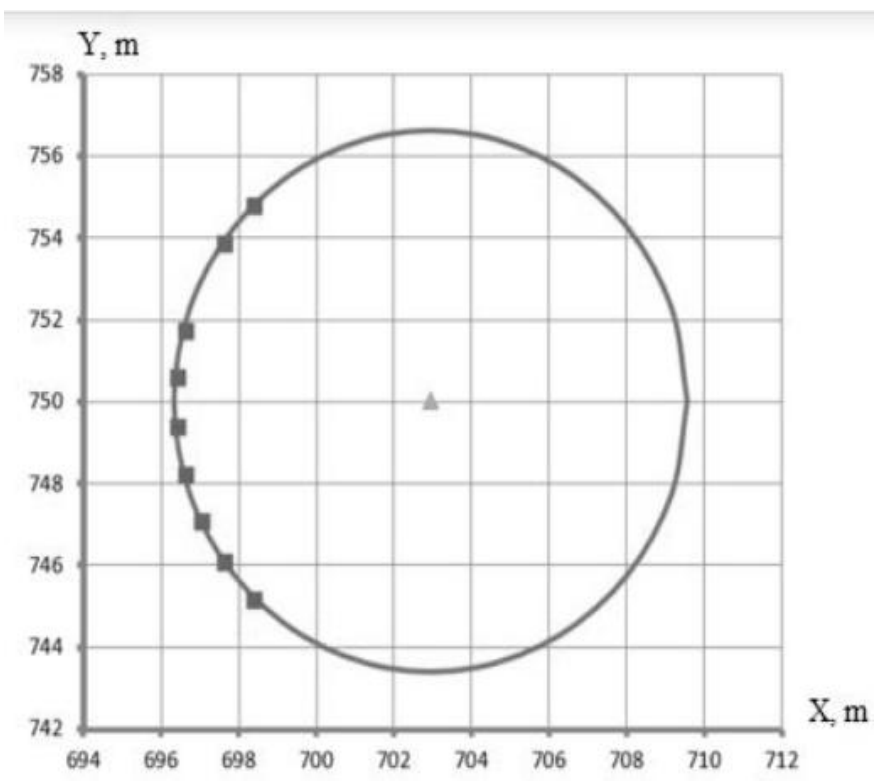

Fig. 2. The built section according to the calculated program parameters.

The squares in Figure 2 show the coordinate points that were obtained as a result of field workwhilescanning the pipe. A triangle is the center of a section. The main advantages of Newton's method can be explained by the fact that, the second partial derivatives of the function, which are responsible for the concavity of the function and allow us to find the smallest value along the curve, are used when expanding the function in the Taylor series. Since the gradient method only requires the value of the first derivatives (geometrically, it is a tangent), the smallest value of the function can be found only at the ends of the line, not along it.

\section{Conclusions}

The article describes the theory of the Newton method of the second order. The method was used to approximate the circumference of the results of measurements of the chimney. The main advantages of the method have been confirmed in solving this problem, namely the high rate of convergence of the method compared with the method of gradient descent with 
a constant pitch, as well as the ability to use rough preliminary values of the parameters for the iterative process.

The main disadvantages of the method are the highly complex computational process (compilation of the Hessian matrix and control of its sign), as well as the difficult preliminary preparation of the problem for solution. However, it is possible to simplify the problem of pre-deployment learning with the use of modern technological achievements, namely with the creation of a variety of programs for automating the computing process. Therefore, in the process of writing this article a program was created to calculate the parameters of a circumference when constructing sections of a chimney. This made it possible to automate a complex computational process and successfully apply the secondorder Newton method. In the future, based on this work, it is planned toinvestigate the subject of alignment of geodetic networks using an objective function other than the leastsquares method using of the second order Newton method.

\section{References}

1. A. A.Kuzin, , V. A. Valkov, \& A. I. Kazantsev, JP Conf. Series1118 (1), 012022 (2018).

2. V. Y. Tsvetkov, \& V. V. Oznamets, Geodeziai Kartografia Littoral monitoring using unmanned aerial vehicle 959(5), 2-10 (2020).

3. S. N. Men'shikov, et al. Journal of Mining InstituteSpatial models developed using laser scanning at gas condensate fields in the northern construction- climatic zone $\mathbf{2 3 8}$, 430-437 (2019).

4. N. S., Kopylova, \& I. P. Starikov, Journal of Physics: Conference Series, Fresh approaches to earth surface modeling. 1015(3) (2018).

5. M. G. Mustafin, , V. A Valkov,. \& A. I. Kazantsev, Procedia Engineering, 189, 729736 (2017)

6. D. V. Beregovoi, et al. Procedia Engineering189, 737-743 (2017)

7. G.V Makarov,. Evaluation of accuracy in search methods of adjustment. Geodesy and cartography 11, 20-22 (1981)

8. D.M. Himmelblau, Applied nonlinear programming. New York: McGraw-Hill (1972).

9. V. I. Mitskevich, Mathematical methods and computer models. Novopolotsk: Polotsk state university (2007).

10. Ch. N. Zheltko. et al. The search method of adjustment and assessment of the accuracy of the unknowns in the least squares method. Krasnodar: Kuban State Technological University (2016).

11. V.A. Kougia, Mathematical modeling in the processing of geodetic measurements. St. Petersburg: St. Petersburg Mining University. (in Russian)

12. M.Ya Bryn,. et al. 2014. Adjustment of geodetic measurements in a parametric way. St. Petersburg: emperor Alexander I St. Petersburg state transport (2007).

13. N.N. Eliseyeva, Topical Issues of Rational Use of Natural ResourcesThe application of search methods for solving optimization problems 1, 346-352 (2019).

14. Gribov, M.G. \&Khachumov, V.M. Autometry Determining the geometric parameters of objects from raster images. 1, 40-49 (2001) 
15. V.G. Baranov, \& N.Yu. Ilyasov, Non-contact measurement of the radius of curvature of spherical surfaces. Abstracts of the International Conference "Pattern Recognition and Image Analysis: New Information Technologies"3 (1), 458-462 (2000).

16. V.S., Gapanovich, I.V. Gapanovich, Methods for solving optimization problems. Tyumen: TII-Tsogu-TIU (2014) 